\title{
A continuum anisotropic damage model with unilateral effect
}

\author{
A. Alliche \\ Sorbonne Universités, UPMC Univ Paris 06, CNRS, UMR 7190, Institut Jean Le Rond d'Alembert, \\ 75005 Paris, France \\ Correspondence to: A. Alliche (abdenour.alliche@upmc.fr)
}

Received: 21 September 2015 - Revised: 16 December 2015 - Accepted: 18 January 2016 - Published: 10 February 2016

Abstract. A continuum damage mechanics model has been derived within the framework of irreversible thermodynamics with internal variables in order to describe the behaviour of quasi-brittle materials under various loading paths. The anisotropic character induced by the progressive material degradation is explicitly taken into account, and the Helmholtz free energy is a scalar function of the basic invariants of the second order strain and damage tensors. The elastic response varies depending on the closed or open configuration of defects. The constitutive laws derived within the framework of irreversible thermodynamics theory display a dissymmetry as well as unilateral effects under tensile and compressive loading conditions. This approach verifies continuity and uniqueness of the potential energy. An application to uniaxial tension-compression loading shows a good adequacy with experimental results when available, and realistic evolutions for computed stresses and strains otherwise.

\section{Introduction}

Most geomaterials and concrete are regarded as isotropic and heterogeneous materials before any mechanical loading at mesoscopic scale. The application of a mechanical loading causes occurence of defects whose directions of propagation depends on the local stress field, Microcracks propagate in a direction normal to tension, but tend to close in the case of compression with possible frictional slip on the lips of discontinuities. Complete crack closure causes a recovery of the material stiffness in the direction of compressive stress, this phenomenon is called unilateral effect (Ramtani et al., 1992; Yazdani and Schreyer, 1988; Torrenti and Djebri, 1990; Krajcinovic, 1989). In addition, under a simple mechanical test one observes that cancelation of loading leads to more or less important irreversible strains (Ortiz, 1985). These effects are caused by frictions at crack closure.

Damage mechanics offer a convenient theoretical tool for describing the complex mechanisms associated with damage and failure processes observed under mechanical loading. Many works are reported concerning damage in concrete and geomaterials (Yazdani and Schreyer, 1988; Ortiz, 1985; Alliche and Dumontet, 2011). The production of nu- merous macroscopic models is mainly imposed by the complexity and the variety of the observed behaviors (Pigeon, 1969; Chaboche, 1993; Rabier, 1989; Marigo, 1985). Most approaches favor the simplicity of the formulation by using a single scalar damage parameter to describe density of defects. More realistic damage model requires a tensorial formulation for a system of defects strongly influenced by the local field of stress. Some models take into account the unilateral effect (Badel et al., 2007; Halm and Dragon, 1998; Desmorat et al., 2007; Challamel et al., 2005; Alliche and Dumontet, 2011) and the dissymmetry between tension and compression.

Therefore, the formulation of any continuum damage model must account for the principal characteristics described previously, which are summarized below:

- degradation of material properties induced by the creation or propagation of defects,

- anisotropic behavior as a consequence of damage,

- dissymmetry in behavior between tension and compression, 
- occurrence of irreversible strains after complete unloading.

Furthermore the potential of free energy must verify the property of continuity. This implies conditions on the expression of the jump of the elastic stiffness tensor in the open and closed configuration of the discontinuities.

The present model takes in account the various characteristics quoted above. The potential of free energy is considered as a scalar function of the strain and damage tensors. The constitutive equations are applied to describe the anisotropic elastic damage behavior of concrete. A confrontation is made with existing experimental measurements (Murakami and Kamiya, 1997).

The model has been implemented in the finite element software Plaxis, in order to investigate the model response under more general stress state conditions, as well as to assess the applicability of the model to geotechnical case studies such as deep underground excavations in rocks.

\section{Model formulation}

\subsection{Tensorial damage variable}

The presence of microdiscontinuities and their evolution in the material structure leads to an alteration of its mechanical properties. These microcracks exist in different forms and at different scales, in particular in heterogeneous brittle media which geomaterials constitute one of the representative classes.

The need for predicting the mechanical response of geomaterials under various loading paths leads to the representation of such defects by a damage variable, which can take a scalar or tensorial character depending on the degree of characterization of defects. For this class of materials, damage can be represented by a distribution of defects depending on the local field of stress and strain.

Anisotropic damage results from a distribution system of $n$ defects. In the case of a configuration of parallel defects, the associated damage parameter can be written as follows:

$\mathbf{D}=D_{i} \boldsymbol{n}_{i} \otimes \boldsymbol{n}_{i}$,

where $D_{i}$ is the defects density for the $i$ th family of microcracks of orientation $n_{i}$. Consequently, for all the defects contained within the microstructure, we obtain by accounting for all the possible orientations:

$\mathbf{D}=\sum_{i} D_{i} \boldsymbol{n}_{i} \otimes \boldsymbol{n}_{i}$

The second order damage tensor in Eq. (2) is symmetric and therefore has three eigenvalues $D_{k}$ associated with three eigenvectors $E_{k}(k=1,2,3)$. Consequently, any system of discontinuities can be represented by $p$ parallel families of microcracks, which can be reduced to three mutually orthogonal defect densities $D_{k}(k=1,3)$ :

$\mathbf{D}=\sum_{k=1}^{3} D_{k} \boldsymbol{n}_{k} \otimes \boldsymbol{n}_{k}$.

\subsection{Thermodynamic potential and state laws}

In the case of an elastic damaged material, the thermodynamic potential of free energy $w$ is a scalar function of two state variables, namely the strain tensor $\boldsymbol{\varepsilon}$ and the damage tensor D:

$w=w(\boldsymbol{\varepsilon}, \mathbf{D})$.

The scalar function in Eq. (4) is taken to be linear with respect to $\mathbf{D}$ in the case of non-interacting defects and quadratic with respect to $\varepsilon$ to express the linear nature of the behavior law for this class of materials. A possible form of the potential of free energy is written below:

$w(\boldsymbol{\varepsilon}, \mathbf{D})=\frac{1}{2} \boldsymbol{\varepsilon}: \mathbb{C}(\mathbf{D}): \boldsymbol{\varepsilon}$,

where $\mathbb{C}(\mathbf{D})$ is the fourth order stiffness tensor of an isotropic material for a given damage state. In particular, for the virgin material prior to damage onset, we have:

$\mathbb{C}(\mathbf{D}=\mathbf{0})=\mathbb{C}_{0}$

Damage can be viewed as a perturbation of the material structure, resulting in a decrease in the potential of free energy. Therefore the potential of the damaged material is considered to be equal to the potential of the undamaged material $w_{0}(\boldsymbol{\varepsilon}, \mathbf{D}=\mathbf{0})$ reduced by the energy associated with damage $w_{D}(\boldsymbol{\varepsilon}, \mathbf{D})$ :

$w(\boldsymbol{\varepsilon}, \mathbf{D})=w_{0}(\boldsymbol{\varepsilon}, \mathbf{D}=\mathbf{0})-w_{D}(\boldsymbol{\varepsilon}, \mathbf{D})$.

\subsection{Identification and explicit formulation of an anisotropic damage model}

\subsubsection{Linear elasticity and continuity conditions}

Macroscopic modeling of damage unilateral effects constitutes an open research field. Several formulations have been proposed to solve the problem of the damage activationdeactivation process, also called unilateral effect (Chaboche, 1992). In a critical paper review, Cormery and Welemane (2002) have examined several existing formulations and displayed some inconsistencies in existing models. In particular, their theoretical investigation has demonstrated that the formulations proposed by Chaboche (1993) and Halm and Dragon (1996), which are based on a spectral decomposition of the potential to represent the damage activationdeactivation process, lead to an unacceptable thermodynamic potential. 
A damage brittle material such as geomaterials exhibit different stiffness in compressive and tensile loading with a kink at the origine (Ramtani et al., 1992; Curnier et al., 1995). For modelling such an unilateral response, we consider an elastic potential energy which is continuously differentiable, and piecewise twice continuously differentiable. Furthermore we assume that for a given damage state $\mathbf{D}$, the strain space $E$ can be partitioned into two half-spaces $E^{-}$(for compression) and $E^{+}$(for tension) by means of a hypersurface $\mathcal{J}$ characterized by a scalar-valued function $\Gamma(\varepsilon)$ :

$$
\begin{array}{r}
\mathcal{J}=\{\boldsymbol{\varepsilon} / \Gamma(\boldsymbol{\varepsilon})=0\} \\
E^{-}=\{\boldsymbol{\varepsilon} \in E / \Gamma(\boldsymbol{\varepsilon})<0\} \\
E^{+}=\{\boldsymbol{\varepsilon} \in E / \Gamma(\boldsymbol{\varepsilon}) \geq 0\}
\end{array}
$$

Let $w(\boldsymbol{\varepsilon}, \mathbf{D})$ designate the continuously differentiable energy function defined over the strain space $E$.

$w^{+}(\boldsymbol{\varepsilon}, \mathbf{D})$ and $w^{-}(\boldsymbol{\varepsilon}, \mathbf{D})$ are twice continuously differentiable energy functions, such that:

$w(\boldsymbol{\varepsilon}, \mathbf{D})= \begin{cases}w^{+}(\boldsymbol{\varepsilon}, \mathbf{D}) & \text { if } \Gamma(\boldsymbol{\varepsilon}) \geq 0 \\ w^{-}(\boldsymbol{\varepsilon}, \mathbf{D}) & \text { if } \Gamma(\boldsymbol{\varepsilon})<0\end{cases}$

$\sigma^{+}(\boldsymbol{\varepsilon}, \mathbf{D})$ and $\sigma^{-}(\boldsymbol{\varepsilon}, \mathbf{D})$ represent the first order derivatives of the energy function over their respective subdomains $E^{+}$ and $E^{-}$:

$\sigma(\boldsymbol{\varepsilon}, \mathbf{D})=\left\{\begin{array}{l}\sigma^{+}(\boldsymbol{\varepsilon}, \mathbf{D})=\frac{\partial w^{+}(\boldsymbol{\varepsilon}, \mathbf{D})}{\partial \boldsymbol{\varepsilon}} \text { if } \Gamma(\boldsymbol{\varepsilon}) \geq 0 \\ \sigma^{-}(\boldsymbol{\varepsilon}, \mathbf{D})=\frac{\partial w^{-}(\boldsymbol{\varepsilon}, \mathbf{D})}{\partial \boldsymbol{\varepsilon}} \text { if } \Gamma(\boldsymbol{\varepsilon})<0\end{array}\right.$

In the same way, $\mathbb{C}^{+}(\mathbf{D})$ and $\mathbb{C}^{-}(\mathbf{D})$ represent the second order partial derivatives with respect to the strain tensor, defined respectively over $E^{+}$and $E^{-}$:

$\mathbb{C}(\mathbf{D})=\left\{\begin{array}{l}\mathbb{C}^{+}(\mathbf{D})=\frac{\partial^{2} w^{+}(\boldsymbol{\varepsilon}, \mathbf{D})}{\partial \boldsymbol{\varepsilon}^{2}} \text { if } \Gamma(\boldsymbol{\varepsilon}) \geq 0 \\ \mathbb{C}^{-}(\mathbf{D})=\frac{\partial^{2} w^{-}(\boldsymbol{\varepsilon}, \mathbf{D})}{\partial \boldsymbol{\varepsilon}^{2}} \text { if } \Gamma(\boldsymbol{\varepsilon})<0\end{array}\right.$

The continuity of the stress-strain response at the transition between the two states of damage (tension and compression) requires the thermodynamic potential $w(\boldsymbol{\varepsilon}, \mathbf{D})$ to be continuously differentiable. Curnier et al. (1995) and Welemane (2002) have demonstrated that the necessary and sufficient condition for $w(\boldsymbol{\varepsilon}, \mathbf{D})$ to be $\mathrm{C}^{1}-$ continuous can be expressed as follows:

$$
\begin{aligned}
{[[\mathbb{C}(\mathbf{D}]]} & =\mathbb{C}^{+}(\mathbf{D})-\mathbb{C}^{-}(\mathbf{D})=\left[\left[\frac{\partial^{2} w}{\partial \boldsymbol{\varepsilon}^{2}}\right]\right]=s \\
& \cdot(\mathbf{D}) \frac{\partial \Gamma(\boldsymbol{\varepsilon})}{\partial \boldsymbol{\varepsilon}} \otimes \frac{\partial \Gamma(\boldsymbol{\varepsilon})}{\partial \boldsymbol{\varepsilon}}, \forall \boldsymbol{\varepsilon} / \Gamma(\boldsymbol{\varepsilon})=0
\end{aligned}
$$

where $[[\mathbb{C}(\mathbf{D}]]$ represents the jump of the mechanical stiffness tensor through the hyperplane $\Gamma(\varepsilon)=0$.

$s$ is a positive scalar quantity. The previous condition (9) expresses the fact that the jump in the elasticity tensor across the interface is normal to the interface.

\subsubsection{Formulation of the elastic damage model}

The most general form of the thermodynamic potential energy $w(\boldsymbol{\varepsilon}, \mathbf{D})$ can be expressed by a combination the invariants of the strain and damage tensors $\boldsymbol{\varepsilon}$ and $\mathbf{D}$ :

$$
\begin{aligned}
w(\boldsymbol{\varepsilon}, \mathbf{D}) & =w_{0}(\boldsymbol{\varepsilon}, \mathbf{D}=\mathbf{0})+\alpha \operatorname{tr}(\mathbf{D})(\operatorname{tr}(\boldsymbol{\varepsilon}))^{2} \\
& +\beta \operatorname{tr}(\mathbf{D}) \operatorname{tr}\left((\boldsymbol{\varepsilon})^{2}\right)+\gamma \operatorname{tr}(\boldsymbol{\varepsilon} \cdot \mathbf{D}) \operatorname{tr}(\boldsymbol{\varepsilon}) \\
& +\delta \operatorname{tr}\left((\boldsymbol{\varepsilon})^{2} \cdot \mathbf{D}\right),
\end{aligned}
$$

where $\alpha, \beta, \gamma$ and $\delta$ are material constant parameters.

According to Eq. (10), the potential of free energy can be expressed in the configuration where $\epsilon$ belongs to the tension subdomain $E^{+}(\Gamma(\boldsymbol{\epsilon})>0)$ :

$$
\begin{aligned}
w^{+}= & w_{0}^{+}+\alpha^{+} \operatorname{tr}(\mathbf{D})(\operatorname{tr}(\boldsymbol{\epsilon}))^{2}+\beta^{+} \operatorname{tr}(\mathbf{D}) \operatorname{tr}\left((\boldsymbol{\epsilon})^{2}\right) \\
& +\gamma^{+} \operatorname{tr}(\boldsymbol{\epsilon} \cdot \mathbf{D}) \operatorname{tr}(\boldsymbol{\epsilon})+\delta^{+} \operatorname{tr}\left(\boldsymbol{\epsilon}^{2} \cdot \mathbf{D}\right) .
\end{aligned}
$$

When $\boldsymbol{\epsilon}$ belongs to the compression subdomain $E^{-}(\Gamma(\boldsymbol{\epsilon})<$ $0)$ :

$$
\begin{aligned}
w^{-} & =w_{0}^{-}+\alpha^{-} \operatorname{tr}(\mathbf{D})(\operatorname{tr}(\boldsymbol{\epsilon}))^{2}+\beta^{-} \operatorname{tr}(\mathbf{D}) \operatorname{tr}\left((\boldsymbol{\epsilon})^{2}\right) \\
& +\gamma^{-} \operatorname{tr}(\boldsymbol{\epsilon} \cdot \mathbf{D}) \operatorname{tr}(\boldsymbol{\epsilon})+\delta^{-} \operatorname{tr}\left(\boldsymbol{\epsilon}^{2} \cdot \mathbf{D}\right) .
\end{aligned}
$$

From Eqs. (11) and (12) we obtain the following expression for the stiffness tensor discontinuity:

$$
\begin{aligned}
{[[\mathbb{C}(\mathbf{D}]]} & =2\left(\alpha^{+}-\alpha^{-}\right) \operatorname{tr}(\mathbf{D}) \mathbf{1} \otimes \mathbf{1}+2\left(\beta^{+}-\beta^{-}\right) \mathbf{1} \bar{\otimes} \mathbf{1} \\
& +\left(\gamma^{+}-\gamma^{-}\right)(\mathbf{1} \otimes \mathbf{D}+\mathbf{D} \otimes \mathbf{1}) \\
& +\left(\delta^{+}-\delta^{-}\right)(\mathbf{1} \bar{\otimes} \mathbf{D}+\mathbf{D} \bar{\otimes} \mathbf{1})
\end{aligned}
$$

By comparing Eqs. (9) and (10), the function $\Gamma(\boldsymbol{\epsilon})=0$ can be identified as:

$\Gamma(\boldsymbol{\epsilon})=\operatorname{tr}(\boldsymbol{\epsilon})$

The function associated with the hyperplane $\mathcal{J}$ is therefore defined by Eq. (14). This implies that the criterion associated with the transition between the tension hyperspace $E^{+}$and the compression hyperspace $E^{-}$, which is expressed for a given state of the damage variable $\mathbf{D}$, depends solely on the sign of $\operatorname{tr}(\boldsymbol{\epsilon})$. This may be illustrated through the analysis of an uniaxial damage loading test, Fig. 1. The solid lines represent typical virgin loading curves under an uniaxial state of tension or compression alone. The dash lines represent the qualitative response of the model during unloading in the tension domain (after reaching a certain amount of damage) and subsequent compression. During crossing of the hyperplane $\Gamma(\boldsymbol{\epsilon})=\operatorname{tr}(\boldsymbol{\epsilon})=0$, the tensorial damage variable $\mathbf{D}$ remains constant, resulting in a null damage rate. 


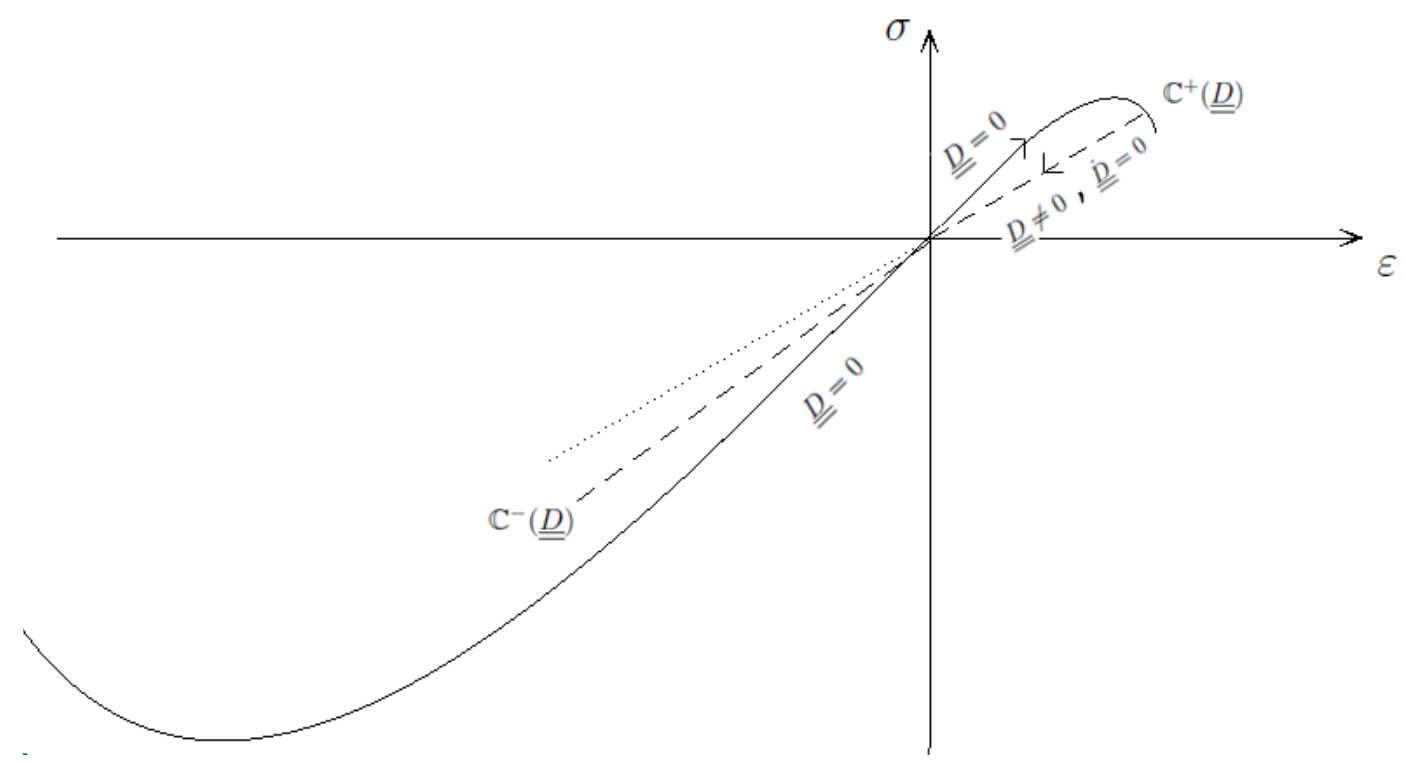

Figure 1. Schematic representation of the transition state between tension and compression loading.

The continuity condition (9) and Eq. (13) imply the following set of relationships, that must be verified by parameters $\alpha^{+}, \alpha^{-}, \beta^{+}, \beta^{-}, \gamma^{+}, \gamma^{-}$and $\delta^{+}, \delta^{-}$:

$\alpha^{+} \neq \alpha^{-}$

$\beta^{+}=\beta^{-}=\beta$

$\gamma^{+}=\gamma^{-}=\gamma$

$\delta^{+}=\delta^{-}=\delta$

Equations (11) and (12) can be summarized in the following expression, which expresses the potential of free energy $w(\boldsymbol{\epsilon}, \mathbf{D})$ in a unified way:

$$
\begin{aligned}
w(\boldsymbol{\epsilon}, \mathbf{D}) & =G_{0} \operatorname{tr}\left(\boldsymbol{\epsilon}^{D} \cdot \boldsymbol{\epsilon}^{D}\right)+\frac{K_{0}}{2}(\operatorname{tr}(\boldsymbol{\epsilon}))^{2} \\
& +\alpha^{+}\left[(\operatorname{tr}(\boldsymbol{\epsilon}))_{+}\right]^{2} \operatorname{tr}(\mathbf{D})+\alpha^{-}\left[(-\operatorname{tr}(\boldsymbol{\epsilon}))_{+}\right]^{2} \operatorname{tr}(\mathbf{D}) \\
& +\beta \operatorname{tr}(\mathbf{D}) \operatorname{tr}\left((\boldsymbol{\epsilon})^{2}\right)+\gamma \operatorname{tr}(\boldsymbol{\epsilon} \cdot \mathbf{D})+\delta \operatorname{tr}\left(\boldsymbol{\epsilon}^{2} \cdot \mathbf{D}\right),
\end{aligned}
$$

where $\boldsymbol{\epsilon}^{D}=\boldsymbol{\epsilon}-\frac{1}{3} \operatorname{tr}(\boldsymbol{\epsilon}) \mathbf{1}$ is the deviatoric strain tensor, and $K_{0}=\frac{3 \lambda_{0}+2 \mu_{0}}{3}$ the compressibility modulus.

The terms in $\alpha^{+}$and $\alpha^{-}$in Eq. (15) reflect the unilateral effect due to possible partial deactivation of defects.

\section{State laws and damage rate evolution}

\subsection{State laws : stress tensor and thermodynamic force}

The macroscopic tensor $\sigma$ is obtained by partial derivation of $w(\boldsymbol{\epsilon}, \mathbf{D})$ with respect to the strain tensor $\boldsymbol{\epsilon}$ :

$$
\begin{aligned}
\boldsymbol{\sigma} & =\frac{\partial w}{\partial \boldsymbol{\epsilon}}=2 G_{0} \boldsymbol{\epsilon}^{D}+K_{0} \operatorname{tr}(\boldsymbol{\epsilon}) \mathbf{1}+2 \alpha^{+}(\operatorname{tr}(\boldsymbol{\epsilon}))_{+} \operatorname{tr}(\mathbf{D}) \mathbf{1} \\
& -2 \alpha^{-}(-\operatorname{tr}(\boldsymbol{\epsilon}))_{+} \operatorname{tr}(\mathbf{D}) \mathbf{1} \\
& +2 \beta \operatorname{tr}(\mathbf{D}) \boldsymbol{\epsilon}+\gamma[\operatorname{tr}(\boldsymbol{\epsilon} \cdot \mathbf{D}) \mathbf{1}+\operatorname{tr}(\boldsymbol{\epsilon}) \mathbf{D}] \\
& +\delta(\boldsymbol{\epsilon} \cdot \mathbf{D}+\mathbf{D} \cdot \boldsymbol{\epsilon}) .
\end{aligned}
$$

The second state law allows to introduce the thermodynamic force associated to the second order damage tensor $\mathbf{D}$ :

$$
\begin{aligned}
\mathbf{Y} & =-\frac{\partial w}{\partial \mathbf{D}}=-\alpha^{+}(\operatorname{tr}(\boldsymbol{\epsilon}))_{+}^{2} \mathbf{1}-\alpha^{-}(-\operatorname{tr}(\boldsymbol{\epsilon}))_{+}^{2} \mathbf{1} \\
& -\beta \operatorname{tr}(\boldsymbol{\epsilon} \cdot \boldsymbol{\epsilon}) \mathbf{1}-\gamma \operatorname{tr}(\boldsymbol{\epsilon}) \boldsymbol{\epsilon}-\delta \boldsymbol{\epsilon} \cdot \boldsymbol{\epsilon}
\end{aligned}
$$

\subsection{Damage criterion}

Experimental tests performed on geomaterials show the existence of an area in the strain space inside which damage is negligible. Initiation or evolution of defects may appear only if the state of strain reaches the limit of this area. We assume the existence of a damage criterion in the space of thermodynamic forces written in the following form:

$F(\mathbf{Y}, \mathbf{D})=\|\mathbf{Y}\|-\chi(\mathbf{D})$,

$\chi(\mathbf{D})$ is a scalar function, taken to be linear with respect to D:

$\chi(\mathbf{D})=a_{1} \operatorname{tr}(\mathbf{D})+a_{0}$, 
where $a_{0}$ and $a_{1}$ are material constants: $a_{0}$ characterizes the initial damage threshold, while $a_{1}$ describes the manner in which the surface evolves with damage.

The choice of the criterion (18) indicates that the model is associated, and damage evolution is assumed to follow the normality rule:

$\dot{\boldsymbol{D}}= \begin{cases}0, & \text { if } F<0, \text { or } \mathrm{F}=0 \text { and } \dot{F}<0 ; \\ \dot{\lambda} \frac{\partial F(\mathbf{Y}, \mathbf{D})}{\partial \mathbf{Y}}, & \text { if } F=0 \text { and } \dot{F}=0 .\end{cases}$

The damage multiplier $\dot{\lambda}$ is determined by the consistency equations $F(\mathbf{Y}, \mathbf{D})=0$ and $\dot{F}(\mathbf{Y}, \mathbf{D})=0$, leading to:

$\dot{\boldsymbol{D}}=\frac{\operatorname{tr}(\mathbf{Y} \cdot \dot{\boldsymbol{Y}})}{a_{1} \operatorname{tr}(\dot{\boldsymbol{Y}})\|\mathbf{Y}\|} \mathbf{Y}$.

From Eq. (21), the positivity of the dissipation $\mathcal{D}$ is immediately verified:

$\mathcal{D}=\mathbf{Y} \cdot \dot{\boldsymbol{D}}$.

\section{Application to uniaxial tension}

The proposed model can be developed analytically and explicitly in the case of uniaxial monotonic tension. Let $\boldsymbol{e}_{1}$ be the direction of tension loading. The stress, strain and damage tensors are given below:

$\boldsymbol{\sigma}=\left(\begin{array}{ccc}\sigma_{1} & 0 & 0 \\ 0 & 0 & 0 \\ 0 & 0 & 0\end{array}\right), \boldsymbol{\epsilon}=\left(\begin{array}{ccc}\epsilon_{1} & 0 & 0 \\ 0 & \epsilon_{2} & 0 \\ 0 & 0 & \epsilon_{3}\end{array}\right)$,

$\mathbf{D}=\left(\begin{array}{ccc}D_{1} & 0 & 0 \\ 0 & D_{2} & 0 \\ 0 & 0 & D_{3}\end{array}\right)$.

We have: $\sigma_{1} \geq 0, \epsilon_{1} \geq 0, \epsilon_{2}=\epsilon_{3} \leq 0$, which implies $D_{2}=$ $D_{3}$, and we consider that $\operatorname{tr}(\boldsymbol{\epsilon}) \geq 0$. Using the previous expressions, constitutive relations (16) simplify in the following way:

$$
\begin{aligned}
\sigma_{1}= & \frac{4 G_{0}}{3}\left(\epsilon_{1}-\epsilon_{2}\right)+K_{0} \operatorname{tr}(\boldsymbol{\epsilon})+2\left[\alpha^{+} \operatorname{tr}(\boldsymbol{\epsilon})+\beta \epsilon_{1}\right] \operatorname{tr}(\mathbf{D}) \\
& +\gamma\left[\operatorname{tr}(\boldsymbol{\epsilon} \cdot \mathbf{D})+\operatorname{tr}(\boldsymbol{\epsilon}) D_{1}\right] \\
\epsilon_{2}= & \frac{\frac{2 G_{0}}{3}-K_{0}-2 \alpha^{+}\left(D_{1}+2 D_{2}\right)-\gamma\left(D_{1}+D_{2}\right)}{\frac{2 G_{0}}{3}+2 K_{0}+2\left(2 \alpha^{+}+\beta\right)\left(D_{1}+2 D_{2}\right)+2(2 \gamma+\delta) D_{2}} \epsilon_{1} .
\end{aligned}
$$

The components of the thermodynamic damage force are obtained from Eq. (17):

$$
\begin{aligned}
& Y_{1}=-\alpha^{+} \operatorname{tr}\left((\boldsymbol{\epsilon})_{+}\right)^{2}-\beta \operatorname{tr}\left(\epsilon^{2}\right)-\gamma \operatorname{tr}(\boldsymbol{\epsilon}) \epsilon_{1}-\delta\left(\epsilon_{1}\right)^{2}, \\
& Y_{2}=Y_{3}=-\alpha^{+} \operatorname{tr}\left((\epsilon)_{+}\right)^{2}-\beta \operatorname{tr}\left(\epsilon^{2}\right)-\gamma \operatorname{tr}(\boldsymbol{\epsilon}) \epsilon_{2}-\delta\left(\epsilon_{2}\right)^{2} .
\end{aligned}
$$

The damage criterion is then expressed as:

$$
F(\mathbf{Y}, \mathbf{D})=\left(Y_{1}^{2}+2 Y_{2}^{2}\right)^{\frac{1}{2}}-a_{1 t} \operatorname{tr}(\mathbf{D})+a_{0 t}
$$

Constant $a_{0 t}$ is identified at the onset of damage by writing:

$F(\mathbf{Y}, \mathbf{D}=\mathbf{0})=\|\mathbf{Y}\|-a_{0 t}=0$

and thus,

$a_{0 t}=\left(\chi_{1 t}^{2}+2 \chi_{2 t}^{2}\right)\left(\epsilon_{1}^{2}\right)_{t}$.

In the previous relation,

$$
\begin{aligned}
& \chi_{1 t}=\alpha^{+}\left(1-2 v_{0}\right)^{2}+\beta\left(1+2 v_{0}^{2}\right)+\gamma\left(1-2 v_{0}\right)+\delta, \\
& \chi_{2 t}=\alpha^{+}\left(1-2 v_{0}\right)^{2}+\beta\left(1+2 v_{0}^{2}\right)+\gamma\left(1-2 v_{0}\right) v+\delta v_{0}^{2},
\end{aligned}
$$

where $\nu_{0}$ is Poisson's ratio for the undamaged material, and $\left(\epsilon_{1}\right)_{t}$ corresponds to the threshold tensile strain at the onset of damage.

Equation (21) gives the components of the damage rate tensor:

$$
\begin{aligned}
& \dot{D}_{1}=\frac{Y_{1} \dot{Y}_{1}+2 Y_{2} \dot{Y}_{2}}{a_{1} \operatorname{tr}(\mathbf{Y})\|\mathbf{Y}\|} Y_{1}, \\
& \dot{D}_{2}=\dot{D}_{3}=\frac{Y_{1} \dot{Y}_{1}+2 Y_{2} \dot{Y}_{2}}{a_{1} \operatorname{tr}(\mathbf{Y})\|\mathbf{Y}\|} Y_{2} .
\end{aligned}
$$

We have identified the five parameters of the model in the case of the experimental compression and numerical tension curves of Murakami and Kamiya (1997). The identification of the model parameters has been performed with the numerical optimization software BIANCA (Biological Analysis of Composite Assemblages), which is based on genetical algorithms (Vincenti et al., 2010). The first step consists in identifying the limit elastic strains in tension and compression directly on the experimental curves. The main objective function is the difference between the experimental axial stress values and their numerical counterparts. The five independent damage model parameters are computed by running a large series of simulation, and minimizing the difference between the axial strain-axial stress curve and the corresponding numerical curve in Murakami and Kamiya (1997) in a least square sense.

The elastic material parameters are taken from Murakami and Kamiya (1997): $E=21.4 \mathrm{GPa}, v=0.2$. The values of the five independent damage parameters are the following ones: $\alpha^{+}=-8.015 \mathrm{GPa}, \beta=-3.001 \mathrm{GPa}, \gamma=11.587 \mathrm{GPa}$, $\delta=-5.425 \mathrm{GPa}, a_{1 t}=0.0195 \mathrm{MPa}$.

Figure 2 illustrates the evolution of the longitudinal stress computed from Eq. (23), the computed stress $\sigma_{1}$ is very close to the evolution obtained by Murakami and Kamiya (1997) using their model, which shows the consistency of the identification procedure.

In Fig. 3, we have reported the evolutions of the longitudinal stiffness modulus $E$ and Poisson's ratio v, computed respectively from Eqs. (23) and (24). As expected, Fig. 3 shows a continuous decrease in Young's modulus and Poisson's ratio starting from the damage threshold strain $\left(\epsilon_{1}=4 \times 10^{-4}\right)$. 


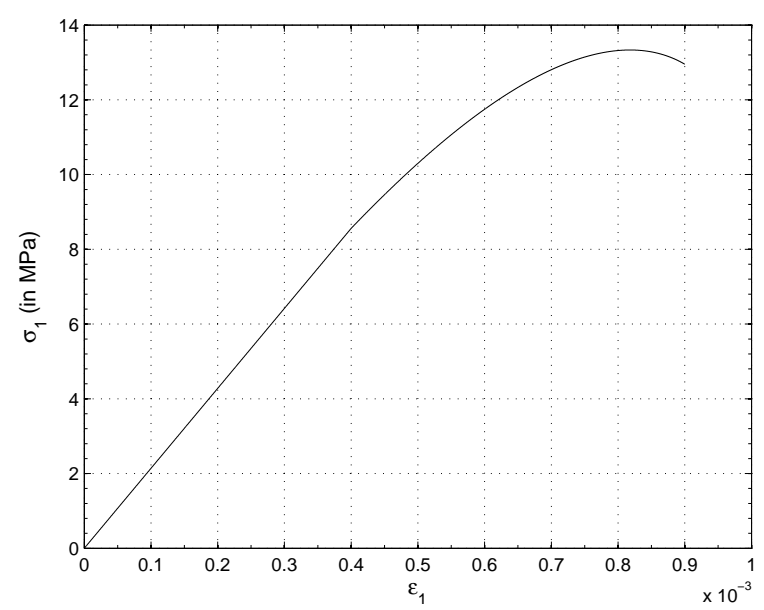

Figure 2. Uniaxial tension. Uniaxial stress-strain curve.

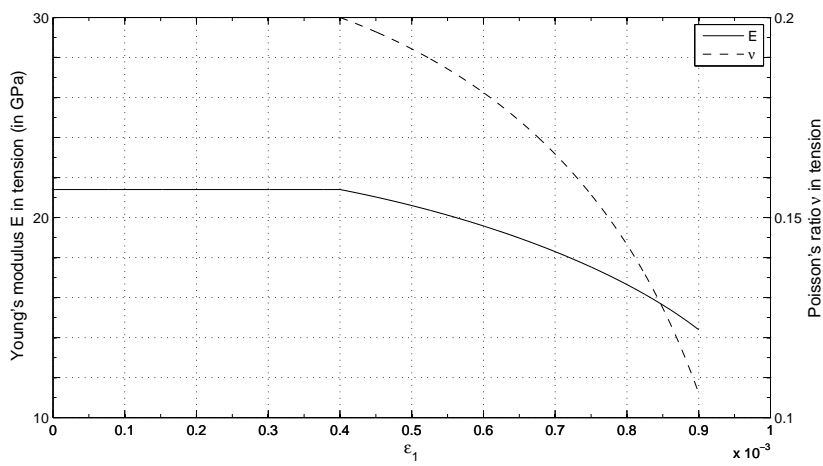

Figure 3. Uniaxial tension. Evolutions of Young's modulus and Poisson's ratio.

\section{Application to uniaxial compression}

Similar to tensile loading, the equations of the proposed model can be developed analytically in the case of uniaxial compression. With $\boldsymbol{e}_{1}$ the direction of the compressive load, the expressions of the stress, strain and damage tensors are identical to the uniaxial tension case, the only difference being that we now consider $\operatorname{tr}(\boldsymbol{\epsilon}) \leq 0$. Similar developments as in Sect. 4 lead to the following set of equations:

$$
\begin{aligned}
\sigma_{1} & =\frac{4 G_{0}}{3}\left(\epsilon_{1}-\epsilon_{2}\right)+K_{0} \operatorname{tr}(\boldsymbol{\epsilon})+2\left[\alpha^{-} \operatorname{tr}(\boldsymbol{\epsilon})+\beta \epsilon_{1}\right] \operatorname{tr}(\mathbf{D}) \\
& +\gamma\left[\operatorname{tr}(\boldsymbol{\epsilon} \cdot \mathbf{D})+\operatorname{tr}(\boldsymbol{\epsilon}) D_{1}\right], \\
\epsilon_{2} & =\frac{\frac{2 G_{0}}{3}-K_{0}-2 \alpha^{-}\left(D_{1}+2 D_{2}\right)-\gamma\left(D_{1}+D_{2}\right)}{\frac{2 G_{0}}{3}+2 K_{0}+2\left(2 \alpha^{-}+\beta\right)\left(D_{1}+2 D_{2}\right)+2(2 \gamma+\delta) D_{2}} \epsilon_{1}, \\
Y_{1} & =-\alpha^{-} \operatorname{tr}\left(-(\boldsymbol{\epsilon})_{+}\right)^{2}-\beta \operatorname{tr}\left(\epsilon^{2}\right)-\gamma \operatorname{tr}(\boldsymbol{\epsilon}) \epsilon_{1}-\delta\left(\epsilon_{1}\right)^{2}, \\
Y_{2} & =Y_{3}=-\alpha^{-} \operatorname{tr}\left(-(\boldsymbol{\epsilon})_{+}\right)^{2}-\beta \operatorname{tr}\left(\epsilon^{2}\right)-\gamma \operatorname{tr}(\boldsymbol{\epsilon}) \epsilon_{2}-\delta\left(\epsilon_{2}\right)^{2} .
\end{aligned}
$$

As in uniaxial tension, the compression parameter $a_{0 c}$ is identified by writing that $F(\mathbf{Y}, \mathbf{D}=\mathbf{0})=\|\mathbf{Y}\|-a_{0 c}=0$ at the

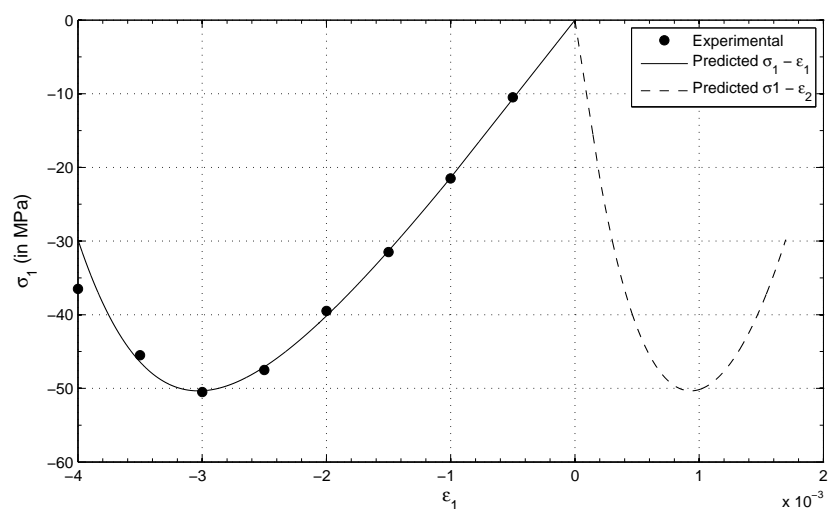

Figure 4. Uniaxial compression. Uniaxial stress-strain curve.

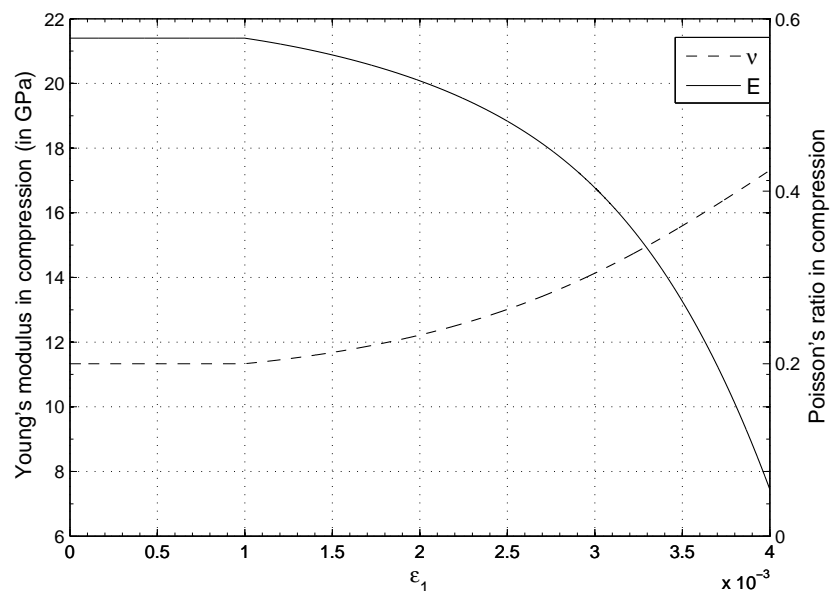

Figure 5. Uniaxial compression. Evolutions of Young's modulus and Poisson's ratio.

onset of damage in compression, resulting in:

$a_{0 c}=\left(\chi_{1 c}^{2}+2 \chi_{2 c}^{2}\right)\left(\epsilon_{1}^{2}\right)_{c}$

where

$\chi_{1 c}=\alpha^{-}(1-2 v)^{2}+\beta\left(1+2 v^{2}\right)+\gamma(1-2 v)+\delta$,
$\chi_{2 c}=\alpha^{-}(1-2 v)^{2}+\beta\left(1+2 v^{2}\right)+\gamma(1-2 v) v+\delta v^{2}$,

and $\left(\epsilon_{1}\right)_{c}$ corresponds to the threshold compressive strain.

Parameters $\beta, \gamma$ and $\delta$ have the same values as for tensile loading. The parameters associated to compression loading have been obtained by applying the optimization procedure to the experimental compression curve: $\alpha^{-}=-0.647 \mathrm{GPa}$ and $a_{1 c}=0.126 \mathrm{MPa}$.

Figures 4 and 5 display respectively the stress-strain, Young's modulus and Poisson's ratio evolutions under uniaxial compression, as predicted by Eqs. (31-32) and a comparison with experimentaldata publishedby Wang and cited by Murakami and Kamiya (1997). Figure 5 shows a decrease in Young's modulus and an increase of Poisson's ratio from 


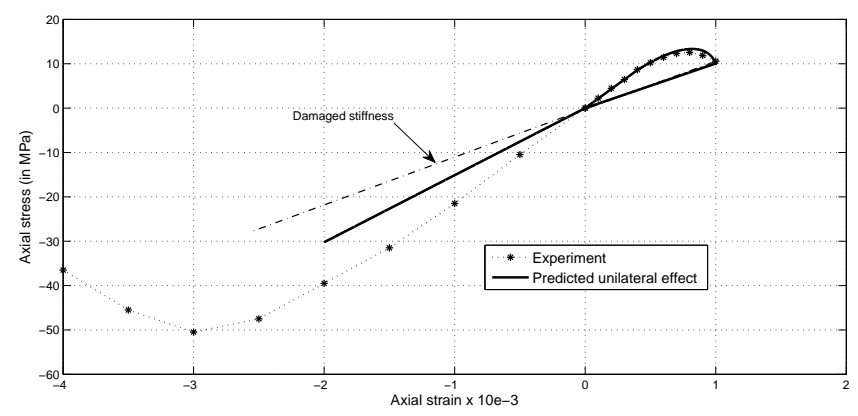

Figure 6. Unilateral effect in tension-compression loading.

the damage threshold strain in compression $\left(\left(\epsilon_{1}\right)_{c}=10^{-3}\right)$. The evolution of these parameters indicates an increase of damage in the transverse direction leading to a diminution of Poisson's ratio. The existence of these microcracks contributes to the weakening of the material stiffness.

Figures 2 and 4 illustrate the dissymmetry existing between the behaviors in tension and compression for this class of material. We observe a ratio of about 4 between the ultimate tensile strength and ultimate compressive stress. This ratio is directly dependent on the values adopted for the parameters of the model.

\section{Unilateral effect}

As mentioned previously, the transition from a tensile loading to a compressive loading leads to partial or complete closure of cracks. This change in microstructure causes a recovery of the stiffness of the damaged material. There are relatively few experimental results published on this subject. Ramtani et al. (1992) showed an unilateral effect with total recovery of the stiffness for the compression phase. The simulation we produce in Fig. 6 clearly indicates that the initial stiffness is not completely recovered, and therefore does not reproduce the behavior described by Ramtani. Nevertheless, we may note that the loading path is quite complex to achieve experimentally. It is likely that some cracks remain open during the compression phase and prevent the total recovery of stiffness.

\section{Conclusion}

A unified model based on continuum damage theory has been developed for quasi-brittle materials such as concrete and rocks. The proposed model relies on a second order tensorial variable to describe anisotropic damage, and has been specifically formulated to be continuously differentiable at the transition between the open and closed cracks configuration. The model has proved to be effective in describing the mechanical behavior of concrete and geomaterials under static loading, more specifically we are able to express:
- the concurrent decrease of Young's modulus and increase of Poisson's ratio with damage development,

- damage-induced anisotropy,

- dissymmetric behavior between tension and compression,

- strain softening behaviour under uniaxial tensioncompression loads.

This model requires the identification of a limited set of five parameters that have been extracted from tensioncompression experiments through an original and systematic identification procedure based on genetic algorithms. A satisfying agreement is obtained between the experimental tension-compression tests and the theoretical simulations.

Future work concerns the investigation of the predictive capacities of the anisotropic damage model with unilateral effects, by confronting Finite Element simulations with available experimental results, including conventional triaxial tests and real case studies.

Edited by: G. Hao

Reviewed by: two anonymous referees

\section{References}

Alliche, A. and Dumontet, H.: Anisotropic model of damage for geomaterials and concrete, Int. J. Numer. Anal. Met., 35, 969979, doi:10.1002/nag.933, 2011.

Badel, P., Godard, V., and Leblond, J.-B.: Application of some damage model to the prediction of the failure of some complex industrial concrete structure, Int. J. Solids Struct., 44, 5848-5874, doi:10.1016/j.ijsolstr.2007.02.001, 2007.

Chaboche, J.-L.: A new unilateral condition for the description of material behavior with anisotropic damage, C.R. Acad. Sci. II, 314, 1395-1401, 1992.

Chaboche, J.-L.: Development of continuum damage mechanics for elastic solids sustaining anisotropic and unilateral damage, Int. J. Damage Mech., 2, 311-329, doi:10.1177/105678959300200401, 1993.

Challamel, N., Lanos, C., and Casandjian, C.: Strain-based anisotropic modelling and unilateral effects, Int. J. Mech. Sci., 47, 459-473, doi:10.1016/j.ijmecsci.2005.01.002, 2005.

Cormery, F. and Welemane, H.: A critical review of some damage models with unilateral effect, Mech. Res. Commun., 29, 391395, doi:10.1016/S0093-6413(02)00262-8, 2002.

Curnier, A., He, Q. C., and Zysset, P.: Conewise linear elastic materials, J. Elasticity, 37, 1-38, doi:10.1007/BF00043417, 1995.

Desmorat, R., Gatuingt, F., and Ragueneau, F.: Nonlocal anisotropic damage model and related computational aspects for quasi-brittle materials, Eng. Fract. Mech., 74, 1539-1560, doi:10.1016/j.engfracmech.2006.09.012, 2007.

Halm, D. and Dragon, A.: A model of anisotropic damage by mesocrack growth; Unilateral effect, Int. J. Damage Mech., 5, 384-402, doi:10.1177/105678959600500403, 1996. 
Halm, D. and Dragon, A.: An anisotropic model of damage and frictional sliding for brittle materials, Eur. J. Mech. A-Solid., 17, 439-460, doi:10.1016/S0997-7538(98)80054-5, 1998.

Krajcinovic, D.: Constitutive equations for damaging materials, J. Appl. Mech., 50, 355-360, doi:10.1115/1.3167044, 1989.

Marigo, J.-J.: Modeling of brittle and fatigue damage for elastic material by growth of microvoids, Eng. Fract. Mech., 21, 861-874, doi:10.1016/0013-7944(85)90093-1, 1985.

Murakami, S. and Kamiya, K.: Constitutive and damage evolution equation of elastic-brittle materials based on irreversible thermodynamics, Int. J. Mech. Sci., 39, 473-486, doi:10.1016/S00207403(97)87627-8, 1997.

Ortiz, M.: A constitutive theory for inelastic behaviour of concrete, Mech. Mater., 4, 67-93, doi:10.1016/0167-6636(85)90007-9, 1985.

Pigeon, M.: The process of crack initiation and propagation in concrete, $\mathrm{PhD}$ thesis, Imperial College, London, 1969.

Rabier, P. J.: Some remarks on damage theory, Int. J. Eng. Sci., 27, 29-54, doi:10.1016/0020-7225(89)90166-3, 1989.
Ramtani, S., Berthaud, Y., and Mazars, J.: Orthotropic behavior of concrete with directional aspects: modeling and experiments, Nucl. Eng. Des., 133, 97-111, doi:10.1016/00295493(92)90094-C, 1992.

Torrenti, J. M. and Djebri, B.: Constitutive laws for concrete: an attempt of comparison, Proceedings of the Second International Conference on Computer Aided Analysis and Design of Concrete Structures, 4-6 April 1990, Zell am See, Austria, 871-882, 1990.

Vincenti, A., Ahamadian, M. R., and Vannucci, P.: BIANCA: a genetic algorithm to solve hard combinatorial optimisation problems in engineering, J. Global Optim., 48, 399-421, doi:10.1007/s10898-009-9503-2, 2010.

Welemane, H.: Une modélisation des matériaux microfissures. Application aux roches et aux bétons, $\mathrm{PhD}$ thesis, Université Lille 1, France, 2002.

Yazdani, S. and Schreyer, H. L.: An anisotropic damage model with dilatation of concrete, Mech. Mater., 7, 231-244 doi:10.1016/0167-6636(88)90022-1, 1988. 